\title{
Association Between Use of Antihyperlipidemic Agents and Chronic Obstructive Pulmonary Disease in Patients with Hyperlipidemia: A Population-Based Retrospective Cohort Study
}

This article was published in the following Dove Press journal:

International Journal of Chronic Obstructive Pulmonary Disease

\author{
Yi-Fen Lei ${ }^{1,2}$ \\ Hsiu-Chen Lin (iD) 3,4 \\ Hsiu-Li Lin (D) 5 \\ Yow-Sheng Uang ${ }^{2}$ \\ Hui-Wen Cheng ${ }^{2}$ \\ Li-Hsuan Wang id ${ }^{2,6}$ \\ 'Department of Pharmacy, Mackay \\ Memorial Hospital, Taipei I0449, Taiwan; \\ ${ }^{2}$ School of Pharmacy, College of \\ Pharmacy, Taipei Medical University, \\ Taipei II03I, Taiwan; ${ }^{3}$ Department of \\ Pediatrics, School of Medicine, College of \\ Medicine, Taipei Medical University, \\ Taipei II03I, Taiwan; ${ }^{4}$ Department of \\ Clinical Pathology, Taipei Medical \\ University Hospital, Taipei I I03I, Taiwan; \\ ${ }^{5}$ Department of Neurology, General \\ Cathay Hospital, New Taipei City 22174, \\ Taiwan; ${ }^{6}$ Department of Pharmacy, Taipei \\ Medical University Hospital, Taipei I I03I, \\ Taiwan
}

Objective: The effect of statins and fibrates on the risk of chronic obstructive pulmonary disease (COPD) remains unclear. The aim of this study was to investigate the effects of statins and fibrates on the risk of COPD in patients with hyperlipidemia.

Patients and Methods: This study involved a retrospective cohort with a follow-up period of 6 years. We identified patients who were diagnosed as having hyperlipidemia between 2000 and 2016 from Taiwan's National Health Insurance Research Database. A Cox proportional hazard model was used to estimate the risk of COPD among different groups. The dose-related effects of statins and fibrates on the risk of COPD were evaluated according to the defined daily dose (DDD).

Results: Patients with hyperlipidemia not using statins and fibrates (group II) had a significantly higher risk of COPD compared with their comparison group, with an adjusted hazard ratio (HR) of 1.091 [95\% confidence interval (CI): 1.034-1.152, $p<0.01$ ]. Dosedependent reduction in the risk of COPD was observed in patients with hyperlipidemia using statins or fibrates compared with patients not using them. Moreover, with an increase in cumulative exposure, a reduced risk of COPD was observed in patients using more than 361 DDDs, with an adjusted HR of 0.474 (95\% CI: 0.401-0.559, $p<0.001$ ). Patients on fibrate monotherapy using more than 541 DDDs were observed to have an adjusted HR of 0.454 (95\% CI: $0.226-0.910, p<0.05$ ) and those on statin monotherapy with over 361 DDDs were noted to have an adjusted HR of 0.583 (95\% CI: $0.459-0.740, p<0.001$ ).

Conclusion: This study demonstrated that an increase in the cumulative exposure of statins and fibrates significantly reduced the risk of COPD in patients with hyperlipidemia, and the risk reduction appeared to be significantly dose dependent.

Keywords: hyperlipidemia, statin, fibrates, chronic obstructive pulmonary disease, cohort study

\section{Introduction}

Chronic obstructive pulmonary disease (COPD) is a growing global health problem that has been among the top 10 causes of death in Taiwan in several years. ${ }^{1}$ COPD significantly influences patients' ability to work and their quality of life, thereby burdening patients' families and society. ${ }^{2}$ COPD is often associated with various comorbidities, including cardiovascular disease (CVD), diabetes, osteoporosis, anxiety, depression, malignancies, chronic renal failure, and infections. ${ }^{2}$ Moreover,
Correspondence: Li-Hsuan Wang School of Pharmacy, College of Pharmacy, Taipei Medical University, $250 \mathrm{Wu}-\mathrm{Hsing}$ St., Taipei II03I, Taiwan

Email shiuan@tmu.edu.tw 
comorbidities of COPD critically affect major outcomes, including quality of life, ${ }^{3}$ rate of acute exacerbation, ${ }^{4}$ and mortality. ${ }^{5}$ Hyperlipidemia is a risk factor for CVD, and most comorbidities are associated with an increased risk of mortality in patients with COPD. Nevertheless, the effect of hyperlipidemia on the clinical outcomes of patients with COPD is complicated and unclear.

Studies have demonstrated that statins may exert "pleiotropic" effects, ${ }^{6}$ which help decrease oxidative stress and inflammation. ${ }^{7}$ In addition, fibrates reduce airway neutrophils and macrophage infiltration, ${ }^{8}$ as well as inflammation, and are considered beneficial for lung disease. ${ }^{9}$ Several studies have revealed that statins provide significant benefits in patients with COPD $;^{10,11}$ however, works published by Criner et $\mathrm{al}^{12}$ in 2014 and Neukamm et $\mathrm{al}^{13}$ in 2015 have evaluated the benefits of statins in the COPD population and indicated no benefits related to exacerbation rates and pulmonary function. The authors surmised that the dose of statins was too low to exhibit significant anti-inflammatory effects, and the short duration of treatment did not yield substantial clinical outcomes, thereby leading to minimal benefits of statins. Given this, we conducted a retrospective cohort study by obtained data from the National Health Insurance (NHI) Database of Taiwan to investigate the risk of COPD in newly diagnosed patients with hyperlipidemia initiated on statins and fibrates, with a further analysis of the effects of statin and fibrate exposure on the risk of COPD.

\section{Patients and Methods}

\section{Data Sources}

This study was a retrospective cohort study. The study samples were retrieved from the Longitudinal Health Insurance Database (LHID), which consisted of data from 2,000,000 enrollees from the population included in Taiwan's NHI program. The NHI program is an insurance system that covers more than $99 \%$ of the national population in Taiwan and provides data for research purposes. No significant differences were observed related to the distribution of age and gender between the patients in the sample group and the original population. Notably, the database contains diagnosis codes, drug prescriptions, hospital visits, detailed clinical and demographic information of all hospital admissions and ambulatory visits. The datasets of NHI related databases have released for academic research purposes since 2000. All data are anonymized and encrypted in consideration of protection for participants' privacy, thus researcher cannot identify individuals. This study was approved by the Joint Institutional Review Board/Ethics Committee (JIRB) of Taipei Medical University (TMU). The certificate number is N201806005.

\section{Study Sample}

For the study cohort, we identified patients who were newly diagnosed with hyperlipidemia (ICD-9-CM codes 272.X, ICD-10-CM codes E78.0-78.5) during an ambulatory care visit between January 1, 2000, and December 31, 2010. We included patients with a three-time diagnosis of hyperlipidemia, and the date when they were first diagnosed was considered to be the diagnosis date. Notably, patients with a three-time diagnosis of hyperlipidemia would increase the validity of the diagnosis. Furthermore, the LHID provides information on medical orders during ambulatory care visits and hospital admissions. We reviewed this data and determined which enrollees had ever filled in prescriptions for statins and fibrates after the diagnosis date. On the basis of these data, we classified patients with hyperlipidemia into the following two groups: those who received statins or fibrates (group I) and those who did not receive statins and fibrates (group II). For patients of group I, the date of statin or fibrate therapy initiation was considered the index date. The study excluded patients who (1) were less than 40 years of age at the time of diagnosis of hyperlipidemia; (2) had less than a three-time diagnosis of hyperlipidemia between 2001 and 2010; (3) had received statins or fibrates before a hyperlipidemia diagnosis; (4) had less than 90 days of statin or fibrate use within 365 days after the first administration of statins or fibrates; (5) had not received statins or fibrates before December 31, 2010; and (6) had been diagnosed as having COPD before the index date. Finally, patients with hyperlipidemia who had received statins or fibrates were enrolled in group I and those who had not used statins and fibrates were enrolled in group II. The date of the first of diagnosis was assigned as the index date for patients of group II. Furthermore, patients without hyperlipidemia not taking any statins or fibrates were selected as the comparison group. Each patient in group II was matched by age, sex, and index year to four patients of the comparison group. All study enrollees were followed up for 6 years or censored at the date of COPD diagnosis.

\section{Dosage of Statins and Fibrates}

Complete information regarding all prescriptions of statins and fibrates was extracted from the NHI prescription database. Data collected included the date of prescription, the 
daily dose, and the number of days supplied. The total dosage of statins or fibrates prescribed during the followup period was calculated for group I. The defined daily dose (DDD) recommended by the World Health Organization was considered for statins $(2 \mathrm{mg}$ /day for pitavastatin, $10 \mathrm{mg} /$ day for rosuvastatin, $20 \mathrm{mg} /$ day for atorvastatin, $45 \mathrm{mg} /$ day for lovastatin, $60 \mathrm{mg} /$ day for fluvastatin, and $30 \mathrm{mg} /$ day for pravastatin and simvastatin) and fibrates $(200 \mathrm{mg} /$ day for fenofibrate and $1200 \mathrm{mg}$ /day for gemfibrate).

\section{Outcome Measurement and Confounding Factors}

Each patient was followed up for 6 years or until patient has a two-time diagnosis of COPD. The primary outcome was the development of COPD, and the secondary outcome was the dose effect of statins and fibrates on the risk of COPD. Notably, COPD was diagnosed according to ICD-9 codes 491, 492, and 496 and ICD-10 codes J41.0, $\mathrm{J} 43.9$, and J44.9. We adjusted the possible confounding factors for the risk of COPD development, including cancer, pneumonia, CVD, hypertension, diabetes, chronic renal disease, osteoporosis, depression, anxiety, infection, gastroesophageal reflux (GERD), bronchiectasis, asthma, tobacco usage, as well as the use of medications such as oral steroids, inhaled steroids, an inhaled $\beta 2$-adrenergic, inhaled corticosteroid and long-acting $\beta$-agonist combination (ICS/LABA), inhaled muscarinic antagonist, and xanthine.

\section{Statistical Analysis}

Statistical analyses were performed using SAS version 9.4 (SAS Institute Inc., Cary, NC, USA). Student's $t$ test and Pearson's chi-squared test were applied to evaluate the intergroup differences related to sociodemographic characteristics such as age and sex as well as comorbidities. Cox proportional hazard regression model was used to compare the risk of COPD between the study groups and the comparison group. Number of diagnosis for COPD among the various DDD groups was evaluated using

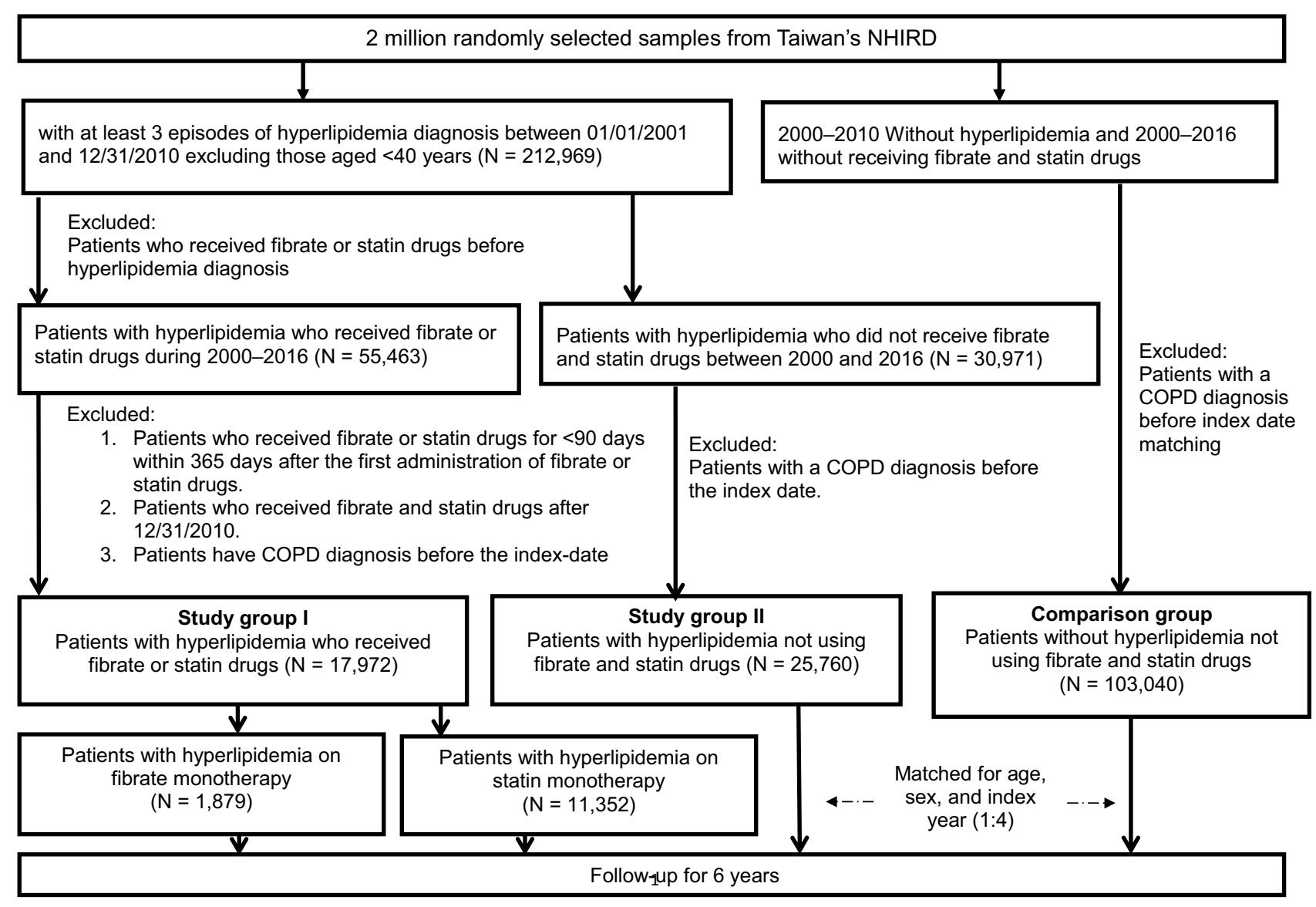

Figure I Flow chart of study population selection.

Abbreviations: COPD, Chronic obstructive pulmonary disease; NHIRD, National Health Insurance Research Database. 
Student's $t$ test. The Kaplan-Meier method and Log rank test were used to examine the differences in 6-year COPD occurrence rates between the study and comparison cohorts. All tests were two sided, and $p$ values less than 0.05 were considered significant.

\section{Results}

\section{Demographic Characteristics}

Overall, 17,972 patients with hyperlipidemia who received statins or fibrates were selected as group I, and 25,760 patients with hyperlipidemia without statin and fibrate use as group II. In addition, 103,040 patients with usage of hyperlipidemia statins or fibrates were selected as the comparison group. Figure 1 illustrates the enrollment of groups I and II as well as the comparison group.

Table 1 lists the demographic characteristics of the three groups. The sex and age distributions were similar among the groups. The mean age of the entire cohort was $56.9 \pm 10.93$ years. The prevalence of comorbid diseases, such as cancer, pneumonia, CVD, hypertension, diabetes, osteoporosis, anxiety, infections, GERD, asthma, tobacco use, and oral steroid use was higher in group I than in the other two groups.

\section{Primary Outcome}

We assessed the crude hazard ratios (HRs) and adjusted HRs for the risk of COPD during the 6-year follow-up period between groups I and II as well as between group II and the comparison group. Patients with hyperlipidemia without statin and fibrate use (group II) had a significantly increased risk of COPD compared with the comparison group, with an adjusted HR of 1.091 [95\% confidence interval (CI): 1.034$1.152, p<0.01]$. The adjusted HR for patients with hyperlipidemia using statins or fibrates (group I) was 0.998 (95\% CI: $0.930-1.070, p>0.05)$ compared with patients with hyperlipidemia not using statins and fibrates (group II), with no significant difference observed (Table 2).

\section{Secondary Outcome}

Upon evaluation of the effects of cumulative DDDs on the risk of COPD, a significant dose-related effect was

Table I Baseline Characteristics of Study Groups and Comparison Group

\begin{tabular}{|c|c|c|c|c|c|}
\hline \multirow[t]{3}{*}{ Variables (\%) } & \multicolumn{5}{|c|}{ Patients with Hyperlipidemia } \\
\hline & \multirow{2}{*}{$\begin{array}{l}\text { Study Group I } \\
N=17,972\end{array}$} & \multirow{2}{*}{$\begin{array}{l}\text { Study Group II } \\
N=25,760\end{array}$} & \multirow{2}{*}{$\begin{array}{l}\text { Comparison Group } \\
N=103,040\end{array}$} & \multirow[t]{2}{*}{$p$ value $^{a}$} & \multirow[t]{2}{*}{$p$ value $^{b}$} \\
\hline & & & & & \\
\hline Age, years (mean $\pm S D$ ) & $56.78 \pm 10.26$ & $56.98 \pm 11.26$ & $56.98 \pm 11.26$ & 0.0572 & 1.0000 \\
\hline Gender/Male (n, \%) & 8,441 (46.97\%) & 12,334 (47.88\%) & $49,336(47.88 \%)$ & 0.0600 & 1.0000 \\
\hline Cancer (n, \%) & $\mathrm{I}, 268(7.06 \%)$ & 2,097 (8.14\%) & 6,277 (6.09\%) & $<0.0001$ & $<0.0001$ \\
\hline Pneumonia (n, \%) & $1,110(6.18 \%)$ & I,39| (5.40\%) & $4,553(4.42 \%)$ & 0.0006 & $<0.0001$ \\
\hline Cardiovascular disease (n, \%) & $13,824(76.92 \%)$ & $16,314(63.33 \%)$ & 32,291 (31.34\%) & $<0.0001$ & $<0.0001$ \\
\hline Hypertension (n, \%) & $12,279(68.32 \%)$ & $13,302(5 \mid .64 \%)$ & 24,361 (23.64\%) & $<0.000$ I & $<0.000$ I \\
\hline Diabetes $(\mathrm{n}, \%)$ & $7,113(39.58 \%)$ & $8,020(31.13 \%)$ & $7,366(7.15 \%)$ & $<0.000$ I & $<0.000$ I \\
\hline Chronic renal disease $(n, \%)$ & $2324(12.93 \%)$ & 3408 (13.23\%) & $477 \mathrm{I}(4.63 \%)$ & 0.3627 & $<0.0001$ \\
\hline Osteoporosis (n, \%) & $7,188(40.00 \%)$ & $9,361(36.34 \%)$ & $25,038(24.30 \%)$ & $<0.000$ I & $<0.0001$ \\
\hline Depression (n, \%) & 961 (5.35\%) & I,347 (3I.I3\%) & $3,199(3.10 \%)$ & 0.5866 & $<0.0001$ \\
\hline Anxiety $(\mathrm{n}, \%)$ & $5,980(33.27 \%)$ & 8,131 (3I.56\%) & $19,249(18.68 \%)$ & 0.0002 & $<0.0001$ \\
\hline Infection (n, \%) & $958(5.33 \%)$ & $\mathrm{I}, 240(4.8 \mathrm{I} \%)$ & $3,690(3.58 \%)$ & 0.0149 & $<0.0001$ \\
\hline GERD $(n, \%)$ & $12,946(72.03 \%)$ & $18,066(70.13 \%)$ & $56,958(55.28 \%)$ & $<0.0001$ & $<0.0001$ \\
\hline Bronchiectasis (n, \%) & $163(0.91 \%)$ & $238(0.92 \%)$ & 721 (0.70\%) & 0.8549 & 0.0002 \\
\hline Asthma (n, \%) & $3,839(21.36 \%)$ & $4,958(19.25 \%)$ & 13,622 (|3.22\%) & $<0.0001$ & $<0.0001$ \\
\hline Tobacco (n, \%) & $303(1.69 \%)$ & $271(1.05 \%)$ & $867(0.84 \%)$ & $<0.000$ I & 0.0012 \\
\hline Oral steroid (n, \%) & II,052 (6I.50\%) & 14,492 (56.26\%) & $51,570(50.05 \%)$ & $<0.000$ I & $<0.0001$ \\
\hline Inhaled steroid (n, \%) & $\mathrm{I}, \mathrm{I} 25$ (6.26\%) & I,535 (5.96\%) & $3,596(3.49 \%)$ & 0.1952 & $<0.0001$ \\
\hline Inhaled $\beta 2$-adrenergic (n, \%) & 491 (2.73\%) & $670(2.60 \%)$ & $1,912(1.86 \%)$ & 0.4014 & $<0.0001$ \\
\hline ICS/LABA (n, \%) & $170(0.95 \%)$ & $23 \mathrm{I}(0.90 \%)$ & $537(0.52 \%)$ & 0.5955 & $<0.0001$ \\
\hline Inhaled muscarinic antagonist ( $\mathrm{n}, \%)$ & $204(1.14 \%)$ & 256 (0.99\%) & $818(0.79 \%)$ & 0.1541 & 0.0016 \\
\hline Xanthine $(\mathrm{n}, \%)$ & $192(1.07 \%)$ & $236(0.92 \%)$ & $744(0.72 \%)$ & 0.1117 & 0.0013 \\
\hline
\end{tabular}

Notes: $p$ value $e^{a}$ study group I (Hyperlipidemia with fibrate or statin drugs) versus study group II (hyperlipidemia without fibrate and statin drugs). $p$ value ${ }^{b}:$ study group II (hyperlipidemia without fibrate or statin drugs) versus comparison group (nonhyperlipidemia without fibrate and statin drugs).

Abbreviations: SD, standard deviation; GERD, gastroesophageal reflux disease; ICS/LABA, inhaled corticosteroid/long-acting $\beta$-agonist combination. 
Table 2 COPD Risk Among Three Groups

\begin{tabular}{|c|c|c|c|}
\hline \multirow[t]{3}{*}{ Results } & \multicolumn{3}{|c|}{ Patients with Hyperlipidemia } \\
\hline & Study Group I & Study Group II & Comparison Group \\
\hline & $N=17,972$ & $N=25,760$ & $N=103,040$ \\
\hline COPD (\%) & $\mathrm{I}, 358$ (7.56\%) & 2,001 (7.77\%) & $6,834(6.63 \%)$ \\
\hline Crude HR (95\% Cl) & - & $1.180(1.123-1.24 I)^{* * *}$ & I \\
\hline Adjusted HR $(95 \% \mathrm{Cl})$ & - & $1.091(1.034-1.152)^{* *}$ & I \\
\hline Crude HR $(95 \% \mathrm{Cl})$ & $0.970(0.905-1.039)$ & 1 & - \\
\hline Adjusted HR $(95 \% \mathrm{Cl})$ & $0.998(0.930-1.070)$ & 1 & - \\
\hline
\end{tabular}

Notes: $*^{* *} p<0.001, * * p<0.01$. The HRs were adjusted for age, gender, cancer, pneumonia, cardiovascular disease, hypertension, diabetes, chronic renal disease, osteoporosis, depression, anxiety, infection, gastroesophageal reflux, bronchiectasis, asthma, tobacco, oral steroid, inhaled steroid, inhaled $\beta 2$-adrenergic, inhaled corticosteroid/long-acting $\beta$-agonist combination, inhaled muscarinic antagonist, xanthine.

Abbreviations: $\mathrm{Cl}$, confidence Interval; COPD, chronic obstructive pulmonary disease; HR, hazard ratio.

observed ( $p$ for trend, $p<0.001$; Table 3). However, when exposure to either statins or fibrates was compared between groups I and II, a reduced risk of COPD was observed with increase in cumulative exposure for those using more than 361-720 DDDs (adjusted HR: 0.474; 95\% CI: 0.401-0.559, $p<0.001$ ), 721-1080 DDDs (adjusted HR: 0.318; 95\% CI:
$0.238-0.425, p<0.001$ ), and $>1081$ DDDs (adjusted HR: 0.193; 95\% CI: $0.125-0.296, p<0.001)$. Compared with group II, the trend analysis of increased cumulative exposure to fibrates revealed a reduced risk of COPD for those using more than 541 DDDs (adjusted HR 0.454; 95\% CI: $0.226-0.910, p<0.05$ ) (Table 4). Similarly, compared

Table 3 Effects of Statin and Fibrate Exposure on COPD Risk

\begin{tabular}{|c|c|c|c|c|c|}
\hline \multirow[t]{3}{*}{ Results } & & \multicolumn{4}{|c|}{ Study Group I (Statin or Fibrate) } \\
\hline & $<360$ DDD & $36 I-720$ DDD & $721-1080$ DDD & $>108 \mid$ DDD & Study Group II \\
\hline & $N=10,200$ & $N=4,278$ & $\mathbf{N}=1,963$ & $\mathbf{N}=\mathbf{I , 5 3 I}$ & $N=25,760$ \\
\hline COPD (\%) & $\mathrm{I}, \mathrm{I} 37$ (II.15\%) & $153(3.58 \%)$ & 47 (2.39\%) & $21(1.37 \%)$ & $2,00 \mathrm{I}(7.77 \%)$ \\
\hline Crude HR & 1.464 & 0.447 & 0.297 & 0.170 & 1 \\
\hline$(95 \% \mathrm{Cl})$ & $(1.362-1.575)^{* * *}$ & $(0.379-0.526)^{* * *}$ & $(0.223-0.397)^{* * *}$ & $(0.111-0.261)^{* * *}$ & \\
\hline Adjusted HR & $\mathrm{I} .444$ & 0.474 & 0.318 & 0.193 & I \\
\hline$(95 \% \mathrm{Cl})$ & $(1.342-1.555)^{* * *}$ & $(0.40 I-0.559)^{* * *}$ & $(0.238-0.425)^{* * *}$ & $(0.125-0.296)^{* * * *}$ & \\
\hline
\end{tabular}

Notes: $* * * p<0.001$. The HRs were adjusted for age, gender, cancer, pneumonia, cardiovascular disease, hypertension, diabetes, chronic renal disease, osteoporosis, depression, anxiety, infection, gastroesophageal reflux disease, bronchiectasis, asthma, tobacco, oral steroid, Inhaled steroid, Inhaled $\beta 2$-adrenergic, Inhaled corticosteroid/ long-acting $\beta$-agonist combination, inhaled muscarinic antagonist, xanthine. $p$ for trend: $<0.001$.

Abbreviations: $\mathrm{Cl}$, confidence Interval; COPD, chronic obstructive pulmonary disease; DDD, the defined daily dose; HR, hazard ratio.

Table 4 Effect of Fibrate Exposure on COPD Risk

\begin{tabular}{|c|c|c|c|c|c|}
\hline \multirow[t]{3}{*}{ Results } & & \multicolumn{4}{|c|}{ Study Group I (Only Fibrate } \\
\hline & $<180$ DDD & I8I-360 DDD & 36I-540 DDD & $>54$ I DDD & Study Group II \\
\hline & $N=1,129$ & $\mathbf{N}=\mathbf{3 3 7}$ & $N=161$ & $N=252$ & $N=25,760$ \\
\hline COPD (\%) & 156 (13.82\%) & $29(8.61 \%)$ & 9 (5.59\%) & 8 (3.17\%) & $2,00 \mathrm{I}(7.77 \%)$ \\
\hline Crude HR & 1.874 & I.108 & 0.707 & 0.396 & I \\
\hline$(95 \% \mathrm{Cl})$ & $(1.593-2.206)^{* * *}$ & $(0.768-1.599)$ & $(0.367-1.359)$ & $(0.198-0.793)^{* *}$ & \\
\hline Adjusted HR & 1.909 & 1.146 & 0.737 & 0.454 & I \\
\hline$(95 \% \mathrm{Cl})$ & $(1.620-2.249) * * *$ & $(0.794-1.655)$ & $(0.383-1.420)$ & $(0.226-0.910)^{*}$ & \\
\hline
\end{tabular}

Notes: $* * * p<0.001, *^{*} p<0.01, *_{p}<0.05$. The HRs were adjusted for age, gender, cancer, pneumonia, cardiovascular disease, hypertension, diabetes, chronic renal disease, osteoporosis, depression, anxiety, infection, gastroesophageal reflux disease, bronchiectasis, asthma, tobacco, oral steroid, inhaled steroid, inhaled $\beta 2$-adrenergic, inhaled corticosteroid/long-acting $\beta$-agonist combination, inhaled muscarinic antagonist, xanthine.

Abbreviations: $\mathrm{Cl}$, confidence Interval; COPD, chronic obstructive pulmonary disease; DDD, the defined daily dose; HR, hazard ratio. 
with group II, an increased cumulative exposure to statins significantly decreased the risk of COPD with exposure to over 361 DDDs (adjusted HR 0.583; 95\% CI: 0.459-0.740, $p<0.001$ ), 541-720 DDDs (adjusted HR 0.273; 95\% CI: 0.179-0.416, $p<0.001$ ), 721-900 DDDs (adjusted HR 0.295; 95\% CI: $0.180-0.483, p<0.001), 901-1080$ DDDs (adjusted HR 0.302; 95\% CI: 0.167-0.547, $p<0.001$ ), 1081-1260 DDDs (adjusted HR 0.398; 95\% CI: $0.198-0.797, p<0.01$ ), and $>1261$ DDDs (adjusted HR 0.099; 95\% CI: 0.037-0.264, $p<0.001$; Table 5).

The Kaplan-Meier curve for the rate of COPD development revealed that patients with hyperlipidemia who did not receive statins or fibrates had the highest cumulative incidence of COPD compared with the other two groups (Figure 2).

\section{Discussion}

This study is the first retrospective cohort study to evaluate the effects of statins and fibrates on COPD risk in patients with hyperlipidemia. An increased risk of COPD was observed in patients with hyperlipidemia compared with those without hyperlipidemia. Nevertheless, patients with hyperlipidemia who received statin and fibrate therapy were noted to have a significantly lower risk of developing COPD within 6 years with an increase in cumulative exposure. Furthermore, a dose-related effect of statins and fibrates on COPD risk was observed.

Notably, both COPD ${ }^{14,15}$ and coronary artery disease ${ }^{16}$ are characterized by low-grade systemic inflammation, which is manifested by increased levels of inflammatory biomarkers. Patients with COPD are at an increased risk of hospitalization and mortality owing to $\mathrm{CVD} .^{17,18}$ Moreover, patients with more severe COPD have higher cardiovascular mortality and morbidity than those with less severe COPD. ${ }^{19}$ Moreover, hyperlipidemia was shown to increase the risk of COPD development. In addition, hyperlipidemia is a major risk factor for CVDs, which are common comorbidities in patients with COPD. ${ }^{20}$ Notably, these ailments have common risk factors, such as smoking, environmental air pollution, age, and CRP level. Notably, dyslipidemia was more prevalent in patients with moderate to severe COPD ${ }^{21-23}$ thus, lipid profile monitoring should be considered in the diagnosis and management of COPD. Therefore, hyperlipidemia was noted to correlate with both CVD and COPD.

Our study findings revealed a clear benefit of statin use in patients with COPD. Statins are lipid-lowering agents that have been widely used to treat hyperlipidemia and

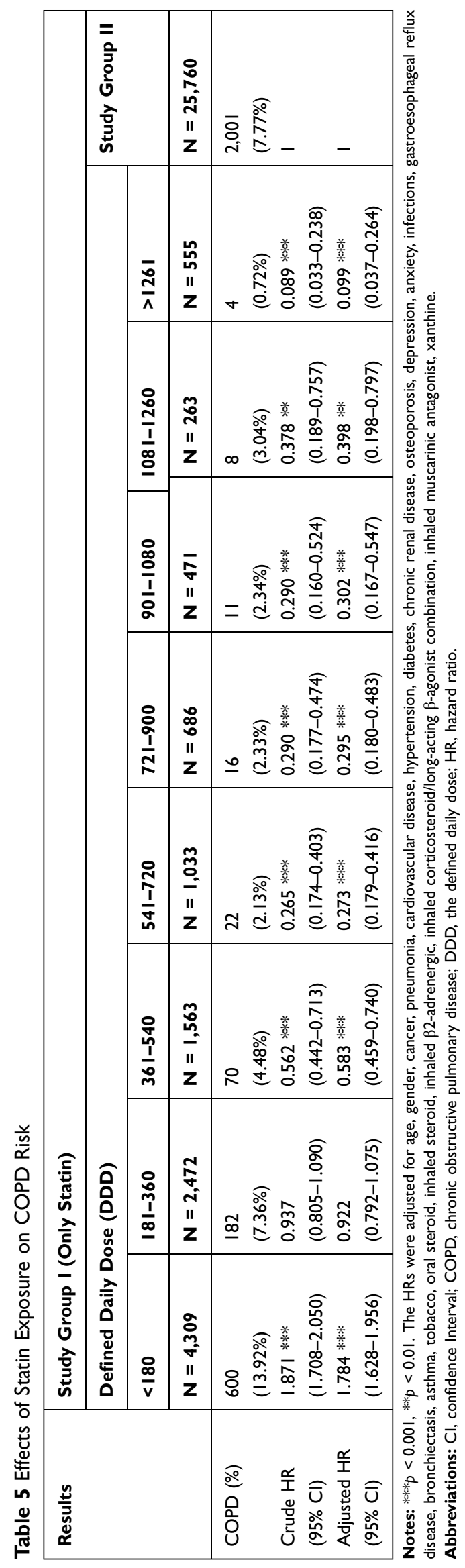


atherosclerotic disease. In addition to its lipid-lowering activity, statins exhibit the protective effects that could be explained by their pleiotropic effects, ${ }^{7}$ such as inhibiting vascular endothelial inflammatory response, stabilizing atheromatous plaque, exerting antithrombotic effects, and improving endothelial function. ${ }^{6}$ Chronic inflammation and oxidation reactions have been noted to play a vital role in the development of CVD. ${ }^{24,25}$ In addition, previous observational studies ${ }^{26-29}$ and systematic meta-analysis reviews ${ }^{30-32}$ have revealed that statin usage exerts antiinflammatory and immunomodulatory effects, lowers the risk of acute exacerbation of COPD, and improves survival after an exacerbation in patients with COPD. Wang et $\mathrm{al}^{33}$ observed that statin use was associated with a reduced risk of COPD exacerbation, with a profound protective effect observed with higher average daily doses of statins. On the other hand, fibrates activate peroxisome proliferator-activated receptor alpha (PPAR- $\alpha$ ), decrease plasma triglycerides and $\mathrm{LDL}$, and elevate $\mathrm{HDL}^{34}$ Functions of PPAR- $\alpha$ receptors in COPD is related to the inhibition of nuclear factor- $\mathrm{BB}(\mathrm{NF}-\kappa \mathrm{B})$ and apolipoprotein A1 (ApoA1) proinflammatory factors. ${ }^{35}$ Notably, PPAR- $\alpha$ activation is considered to be due to their ability to downregulate proinflammatory gene expression, and inflammatory cell functions. ${ }^{36}$ However, most of these published studies have inherent methodological limitations owing to their retrospective or population-based analyses. Therefore, prospective interventional trials are warranted that can precisely assess the effects of statins and fibrates on clinically relevant outcomes in patients with COPD.

Nonetheless, this study had several noteworthy strengths. First, Taiwan's NHI is a large, populationbased database that includes data from a longitudinal cohort. The nationwide LHID provided an excellent resource and opportunity to explore the relationship between COPD risk and the use of statins and fibrates. Second, we only included patients newly diagnosed as having hyperlipidemia without any prior COPD, with at least three consecutive time points of hyperlipidemia diagnosis. Therefore, we could avoid the influence of unknown

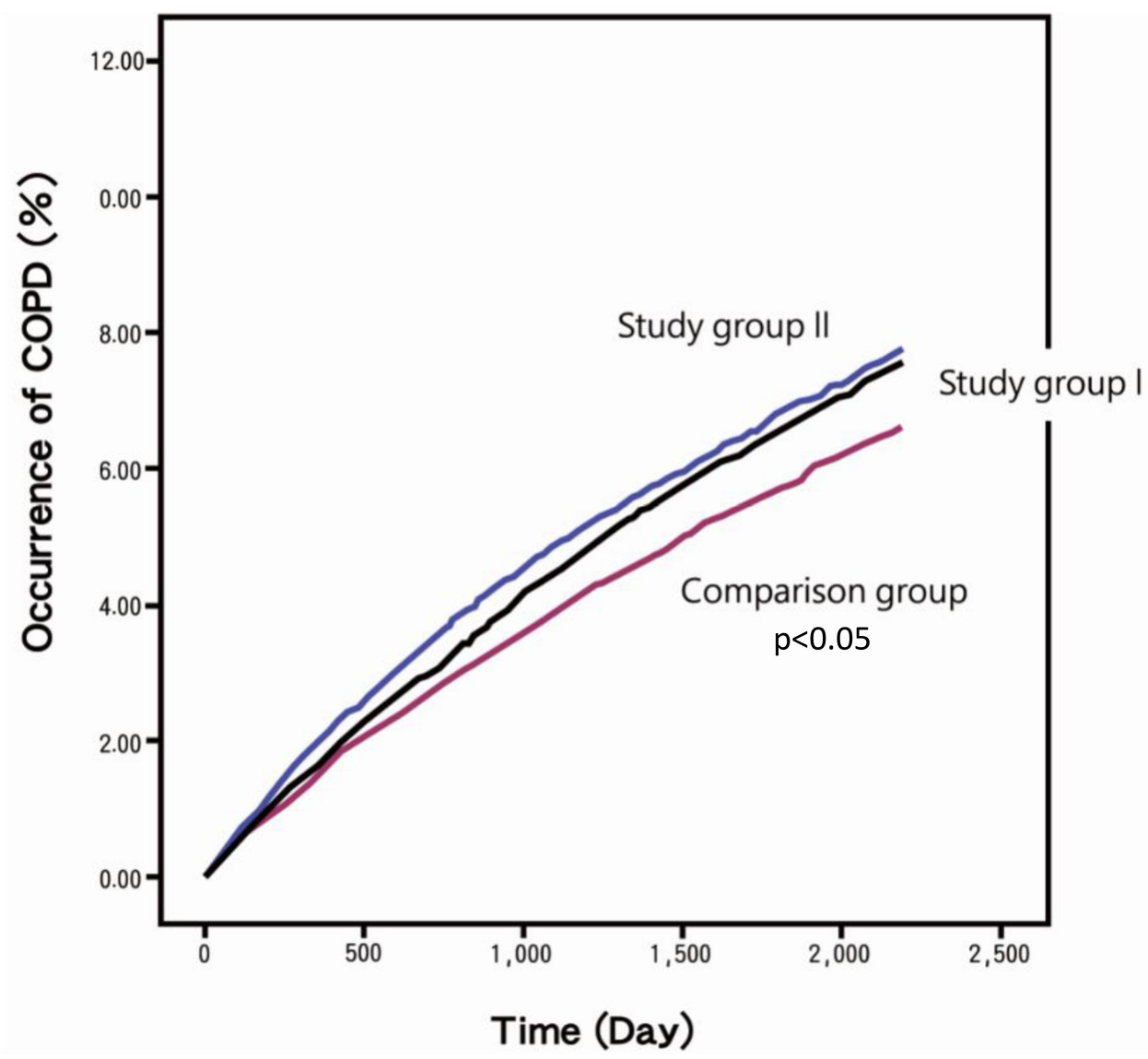

Figure 2 Kaplan-Meier curve of the cumulative occurrence of COPD among three groups. Study group I, hyperlipidemic patients receiving statin or fibrate drugs; study group II, patients with hyperlipidemia not receiving statin and fibrate drugs; comparison group, patients without hyperlipidemia not receiving statin and fibrate drugs. 
treatment histories before the study and increase the accuracy of the diagnosis. Third, potential confounding factors for COPD were considered in the regression models. These factors included age, gender, cancer, pneumonia, CVD, hypertension, diabetes, chronic renal disease, osteoporosis, depression, anxiety, infection, GERD, bronchiectasis, asthma, as well as the use of tobacco, oral steroids, inhaled steroids, inhaled beta-2-adrenergic, ICS/LABA, an inhaled muscarinic antagonist, and xanthine. Last, a further classification of statins and fibrates users based on the DDD used by patients revealed an association between larger doses of statins and fibrates and a more significant reduction of COPD risk.

Nevertheless, this study had some limitations, including the use of an administrative database that lacked records of patients' genetic factors, dyslipidemia phenotype, air pollution levels, and nonprescription medication use. Hence, the effects of these factors could not be evaluated. Moreover, the LHID is a secondary database, and thus, details of patients' disease severity could not be obtained, thereby impeding the stratification of patients based on their lipid levels.

Our findings indicated that statins and fibrates could decrease the risk of COPD in a dose-dependent manner. Nevertheless, further studies are warranted to determine whether statins and fibrates have the potential to be used clinically as preventive agents against COPD.

\section{Conclusion}

Patients with hyperlipidemia have an increased risk of COPD. However, this risk can be decreased in patients with hyperlipidemia using statins or fibrates with an increase in the cumulative dose exposure. Furthermore, COPD risk reduction in patients with hyperlipidemia using statins or fibrates is significantly dose-dependent.

\section{Acknowledgments}

This manuscript was edited by Wallace Academic Editing.

\section{Disclosure}

The authors have no competing interests to disclose.

\section{References}

1. Taiwan COPD Management Guideline. Taiwan Society of Pulmonary and Critical Care Medicine/Health Promotion Administration, Ministry of Health and Welfare; 2019.

2. Global strategy for the diagnosis, management, and prevention of chronic obstructive pulmonary disease. 2020 report. Available from: https://goldcopd.org/wp-content/uploads/2019/11/GOLD-2020REPORT-ver1.0wms.pdf Accessed January 31, 2020.
3. van Manen JG, Bindels PJ, Dekker FW, et al. The influence of COPD on health-related quality of life independent of the influence of comorbidity. J Clin Epidemiol. 2003;56:1177-1184. doi:10.1016/ S0895-4356(03)00208-7

4. Terzano C, Conti V, Di Stefano F, et al. Comorbidity, hospitalization, and mortality in COPD: results from a longitudinal study. Lung. 2010;188(4):321-329. doi:10.1007/s00408-009-9222-y

5. Sin DD, Anthonisen NR, Soriano JB, Agusti AG. Mortality in COPD: role of comorbidities. Eur Respir J. 2006;28:1245-1257. doi:10.1183/09031936.00133805

6. Liao JK, Laufs U. Pleiotropic effects of statins. Annu Rev Pharmacol Toxicol. 2005;45:89-118. doi:10.1146/annurev.pharmtox.45.120403. 095748

7. McFarlane S, Muniyappa R, Francisco R, Sowers J. Pleiotropic effects of statins: lipid reduction and beyond. $J$ Clin Endocrinol Metab. 2002;87(4):1451-1458. doi:10.1210/jcem.87.4.8412

8. Belvisi MG, Hele DJ. Peroxisome proliferator-activated receptors as novel targets in lung disease. Chest. 2008;134:152-157.

9. Becker J, Delayre-Orthez C, Frossard N, Pons F. Regulation of inflammation by PPARs: a future approach to treat lung inflammatory diseases? Fundam Clin Pharmacol. 2006;20:429-447. doi:10.1111/ j.1472-8206.2006.00425.x

10. Huang CC, Chan WL, Chen YC, et al. Statin use and hospitalization in patients with chronic obstructive pulmonary disease: a nationwide population-based cohort study in Taiwan. Clin Ther. 2011;33:1365-1370. doi:10.1016/j.clinthera.2011.08.010

11. Li W-F, Huang Y-Q, Huang C, Feng Y-Q. Statins reduce all-cause mortality in chronic obstructive pulmonary disease: an updated systematic review and meta-analysis of observational studies. Oncotarget. 2017;8:73000.

12. Criner GJ, Connett JE, Aaron SD, et al. Simvastatin for the prevention of exacerbations in moderate-to-severe COPD. $N$ Engl $J$ Med. 2014;370:2201-2210. doi:10.1056/NEJMoa1403086

13. Neukamm A, Høiseth AD, Einvik G, et al. Rosuvastatin treatment in stable chronic obstructive pulmonary disease (RODEO): a randomized controlled trial. $J$ Intern Med. 2015;278:59-67. doi:10. 1111/joim. 12337

14. Stolz D, Christ-Crain M, Morgenthaler NG, et al. Copeptin, C-reactive protein, and procalcitonin as prognostic biomarkers in acute exacerbation of COPD. Chest. 2007;131:1058-1067. doi:10.1378/chest.06-2336

15. Gan WQ, Man SF, Senthilselvan A, Sin DD. Association between chronic obstructive pulmonary disease and systemic inflammation: a systematic review and a meta-analysis. Thorax. 2004;59:574-580. doi:10.1136/thx.2003.019588

16. Danesh J, Wheeler JG, Hirschfield GM, et al. C-reactive protein and other circulating markers of inflammation in the prediction of coronary heart disease. $N$ Engl $J$ Med. 2004;350:1387-1397. doi:10.1056/NEJMoa032804

17. Sidney S, Sorel M, Quesenberry CP Jr, et al. COPD and incident cardiovascular disease hospitalizations and mortality: kaiser permanente medical care program. Chest. 2005;128:2068-2075. doi:10.1378/chest.128.4.2068

18. Curkendall SM, DeLuise C, Jones JK, et al. Cardiovascular disease in patients with chronic obstructive pulmonary disease, Saskatchewan Canada cardiovascular disease in COPD patients. Ann Epidemiol. 2006;16:63-70. doi:10.1016/j.annepidem.2005.04.008

19. Curkendall SM, Lanes S, de Luise C, et al. Chronic obstructive pulmonary disease severity and cardiovascular outcomes. Eur J Epidemiol. 2006;21:803-813. doi:10.1007/s10654-006-9066-1

20. Akhabue E, Thiboutot J, Cheng JW, et al. New and emerging risk factors for coronary heart disease. Am J Med Sci. 2014;347 (2):151-158. doi:10.1097/MAJ.0b013e31828aab45

21. Ritabrata M, Subinay D, Mrinal P, et al. Lipid profile status in chronic obstructive pulmonary disease and association with interleukin $8 . \mathrm{Br}$ J Med Med Res. 2015;9. 
22. Zafirova-Ivanovska B, Stojkovikj J, Dokikj D, et al. The level of cholesterol in COPD patients with severe and very severe stage of the disease. Maced J Med Sci. 2016;4:277. doi:10.3889/oamjms.2016. 063

23. Jain B, Pasari N, Songra A, Bajpai A. The lipid profile parameter in chronic obstructive pulmonary disease patients and correlation with severity of disease. J Cardio Thor Med. 2017;5:564-568.

24. Agustí A, Edwards LD, Rennard SI, et al. Persistent systemic inflammation is associated with poor clinical outcomes in COPD: a novel phenotype. PLoS One. 2012;7:e37483. doi:10.1371/journal. pone.0037483

25. Grundy SM, Stone NJ, Bailey AL, et al. 2018 AHA/ACC/AACVPR/ AAPA/ABC/ACPM/ADA/AGS/APhA/ASPC/NLA/PCNA guideline on the management of blood cholesterol: a report of the American College of Cardiology/American Heart Association Task Force on Clinical Practice Guidelines. J Am Coll Cardiol. 2019;73:24. doi:10.1016/j.jacc.2018.11.003

26. Alexeeff SE, Litonjua AA, Sparrow D, Vokonas PS, Schwartz J. Statin use reduces decline in lung function: VA normative aging study. Am J Respir Crit Care Med. 2007;176:742-747. doi:10.1164/ rccm.200705-6560C

27. Blamoun A, Batty G, DeBari V, et al. Statins may reduce episodes of exacerbation and the requirement for intubation in patients with COPD: evidence from a retrospective cohort study. Int $J$ Clin Pract. 2008;62:1373-1378. doi:10.1111/j.1742-1241.2008.01731.x

28. Frost FJ, Petersen H, Tollestrup K, Skipper B. Influenza and COPD mortality protection as pleiotropic, dose-dependent effects of statins. Chest. 2007;131:1006-1012. doi:10.1378/chest.06-1997
29. Keddissi JI, Younis WG, Chbeir EA, et al. The use of statins and lung function in current and former smokers. Chest. 2007;132:1764-1771. doi:10.1378/chest.07-0298

30. Li W-F, Huang Y-Q, Huang C, Feng Y-Q. Statins reduce all-cause mortality in chronic obstructive pulmonary disease: an updated systematic review and meta-analysis of observational studies. Oncotarget. 2017;8(42):73000. doi:10.18632/oncotarget.20304

31. Raymakers AJ, Sadatsafavi M, Sin DD, De Vera MA, Lynd LD. The impact of statin drug use on all-cause mortality in patients with COPD: a population-based cohort study. Chest. 2017;152:486-493. doi:10.1016/j.chest.2017.02.002

32. Zhang W, Zhang Y, Li C-W, et al. Effect of statins on COPD: a meta-analysis of randomized controlled trials. Chest. 2017;152:1159-1168. doi:10.1016/j.chest.2017.08.015

33. Wang MT, Lo YW, Tsai CL, et al. Statin use and risk of COPD exacerbation requiring hospitalization. Am J Med. 2013;126 (7):598-606. doi:10.1016/j.amjmed.2013.01.036

34. Packard CJ. Management of hypertriglyceridemia: fibrate clinical overview. Eur Heart J. 1998;19(supplA):A62-A65.

35. Belvisi MG, Hele DJ. Peroxisome proliferator-activated receptors as novel targets in lung disease. Chest. 2008;134:52-57.

36. Drost EM, Poland CA, Donaldson K, MacNee W. Diminished peroxisome proliferator-activated receptor (PPAR) regulation as a potential mechanism for the persistent inflammation in chronic obstructive pulmonary disease. Eur Respir Rev. 2006;15:211-212. doi:10.1183/09059180.00010124

\section{Publish your work in this journal}

The International Journal of COPD is an international, peer-reviewed journal of therapeutics and pharmacology focusing on concise rapid reporting of clinical studies and reviews in COPD. Special focus is given to the pathophysiological processes underlying the disease, intervention programs, patient focused education, and self management protocols. This journal is indexed on PubMed Central, MedLine and CAS. The manuscript management system is completely online and includes a very quick and fair peer-review system, which is all easy to use. Visit http://www.dovepress.com/testimonials.php to read real quotes from published authors. 